\title{
Lewis(y) antigen-mediated positive feedback loop induces and promotes chemotherapeutic resistance in ovarian cancer
}

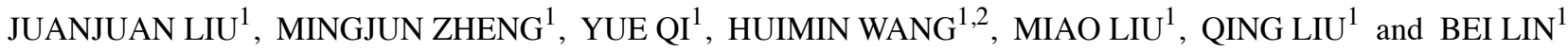 \\ ${ }^{1}$ Department of Obstetrics and Gynecology, China Medical University Shengjing Hospital, Shenyang, Liaoning 110004; \\ ${ }^{2}$ Department of Gynecology, Liaoning Cancer Hospital, Shenyang, Liaoning 110000, P.R. China
}

Received March 9, 2018; Accepted June 25, 2018

DOI: 10.3892/ijo.2018.4496

\begin{abstract}
The present study aimed to investigate the association between Lewis(y) antigen and chemoresistance in ovarian cancer and to elucidate the underlying molecular mechanisms. Lewis(y) expression in chemoresistant ovarian cancer tissues and cells was detected by immunohistochemistry. $\alpha 1,2$-fucosyltransferase (FUT1) expression in different ovarian cancer chemotherapy-resistant cells was analyzed by reverse transcription-quantitative PCR (RT-qPCR). Genes differentially expressed in the chemoresistant and sensitive groups were screened using a gene chip followed by validation using RT-qPCR and western blot analysis. We found that Lewis(y) and FUT1 expression in ovarian cancer cells was significantly increased following the induction of drug resistance. The positive expression rate and intensity of Lewis(y) in ovarian cancer chemoresistant tissues were also significantly higher than those in the sensitive group. Compared with the non-resistant cell lines, the differentially expressed genes were mainly enriched in the terms related to the transmembrane receptor protein tyrosine kinase signaling pathway and positive regulation of cell proliferation. Interaction network analysis predicted genes participating in the regulation of apoptotic processes. The highly differential expression of Annexin A4 (ANXA4), BCL2 interacting killer $(B I K)$, transmembrane $4 \mathrm{~L}$ six family member 4 (TM4SF4) and pleckstrin homologylike domain family A member 1 (PHLDA1) was validated using RT-qPCR in ovarian cancer cell lines. Finally, ANXA4 expression was increased at both the mRNA and protein level in the drug-resistant cells, and in addition, ANXA4 contained a Lewis(y) structure. The expression of Bcl-2 and other antiapoptotic proteins increased with the increase of Lewis $(y)$ expression. After blocking Lewis(y) using an antibody, the
\end{abstract}

Correspondence to: $\mathrm{Dr}$ Bei Lin, Department of Obstetrics and Gynecology, China Medical University Shengjing Hospital, 36 Sanhao Street, Heping, Shenyang, Liaoning 110004, P.R. China E-mail: linbei88@hotmail.com

Key words: Lewis(y) antigen, chemoresistance, positive feedback control loop, signaling pathway network, ovarian cancer expression of the involved signaling pathway and apoptosisrelated proteins decreased significantly. These findings provide strong evidence that Lewis(y) is a component of the structure of the ANXA4 membrane protein. Its overexpression can abnormally activate signaling pathways and regulate the expression of a number of factors, forming a positive feedback loop to induce the chemoresistance of ovarian cancer cells, and ultimately promoting the progression of ovarian cancer.

\section{Introduction}

Ovarian cancer is a gynecological cancer with a high mortality rate. The disease has an occult onset, and approximately $75 \%$ of patients with ovarian cancer have progressed to the advanced stages of the disease by the time of diagnosis. A common treatment for ovarian cancer is cytoreductive surgery, which is surgical removal of the tumor combined with platinum and paclitaxel-based chemotherapy. Although the sensitivity rate of ovarian cancer to paclitaxel and platinum reaches $80 \%$ and the use of chemotherapy has significantly improved overall survival, due to drug resistance, up to $70 \%$ of patients will eventually relapse; the 5-year survival rate remains stagnant at approximately $30 \%(1,2)$. Chemoresistance is a major challenge in the treatment of ovarian cancer. The exact mechanisms responsible for chemoresistance are not yet fully understood, despite extensive research in this area $(3,4)$.

In recent years, the association between glycoconjugates and drug resistance has attracted increasing attention. Glycoconjugates are important components of the cell membrane, and the structure and length of the sugar chain of complex carbohydrates has been found to be altered in cancer cells (5). In ovarian cancer, such changes mainly occur in type II sugar chains, such as Lewis(y). Lewis(y) antigen is a bi-fucosyl containing an oligosaccharide chain in which the fucosylation of the end of the sugar chain is catalyzed by specific $\alpha 1,2$-fucosyltransferase (FUT1). In our previous studies, we transferred the human FUT1 gene into the ovarian cancer cell line, RMG-I, to establish the stably-expressing high-Lewis(y) ovarian cancer cell line, RMG-I-H. We found that the RMG-I-H cells not only exhibited a stronger proliferative and invasive ability, but also exhibited reduced sensitivity to common chemotherapeutic drugs such, as carboplatin, 5-fluorouracil (5-FU) and paclitaxel (6-9). We previously used immunohistochemical staining to examine ovarian cancer 
tissue samples, and found that more sugar chain antigens and cell membrane receptors, including CD44, CD147, human epididymis protein 4 (HE4) and integrin $\alpha 5 \beta 1$ were expressed in chemoresistant ovarian cancer tissues (10-13). These findings suggest that Lewis(y) may be an independent risk factor for chemotherapeutic resistance in ovarian cancer.

The progression from normal ovarian epithelial cells to cancer cells and further to chemotherapy-resistant cells is a complex process, in which cell behaviors, such as proliferation and apoptosis are extensively altered due to gene mutation and related changes in the regulatory mechanism. Therefore, the present study aimed to elucidate the mechanisms responsible for the chemoresistance of ovarian cancer associated with the Lewis(y) antigen. We used a gene chip assay to identify differentially expressed genes (DEGs) between the resistant group and the sensitive group and predicted involved pathways by bioinformatics analysis. The findings were validated at both the mRNA and protein level. This study provides valuable targets for the targeted therapy and for the molecular diagnosis of ovarian cancer.

\section{Materials and methods}

Ethics statement. Samples were fully encoded to protect patient confidentially. The study and its protocols were approved by the Research Ethics committees of Shengjing Hospital Affiliated with China Medical University (2013PS66K). The ethics committee waived the need for patient consent, as the patient information was withheld.

Patient specimens. All tissue samples used in this study (including the colon cancer sample) were obtained from the tissue bank at Shengjing Hospital of China Medical University. Between May, 2006 and July, 2010, 92 ovarian cancer patients were treated at the Department of Gynecology of Shengjing Hospital of China Medical University and were categorized according to the National Comprehensive Cancer Network (NCCN) guidelines, which uses the following definitions: Recurrence during the chemotherapy period (paclitaxel and carboplatin) or within 6 months after the end of chemotherapy is defined as drug resistance; recurrence between 6-12 months after the end of chemotherapy is defined as partial sensitivity; and recurrence beyond 12 months after the end of chemotherapy or no recurrence is defined as sensitivity. Based on these criteria, we divided all patients into the resistant group $(n=37)$ and sensitive group $(n=55)$. Lewis $(y)$ expression in the ovarian cancer tissues was detected by immunohistochemistry and its association with the chemoresistance status of the patients was examined. All the cases are primary, the information and follow-up data are complete, and chemical treatment was not used in any of the patients prior to surgery.

Materials. The following reagents were purchased from commercial sources: Mouse anti-human Lewis(y) monoclonal antibody (cat. no. F3, ab3359) from Abcam (Cambridge, UK); carboplatin as an apoptosis-inducing factor from Shandong Qilu Pharmaceutical Co., Ltd. (Jinan, China); DMEM and fetal bovine serum from HyClone (Logan, UT, USA); the streptavidin-biotin-peroxidase (SP) test kit from Boshide
Biotech Co. (Wuhan, China). The protein content in the cell lysates was measured by BCA assay (Beyotime Institute of Biotechnology, Jiangsu, China); HRP-labeled secondary antibodies (goat anti-mouse IgG-HRP, cat. no. sc-2005, 1:2,000; goat anti-rabbit IgG-HRP, cat. no. sc-2004, 1:2,000) were purchased from Santa Cruz Biotechnology, Inc. (Dallas, TX, USA). For immunoblot analysis, the following antibodies were used: Annexin A4 (ANXA4) (cat. no. sc-28827, 1:200), Bax (cat. no. sc-20067, 1:500), p-Bad (cat. no. sc-166932, 1:500), Bcl-2 (cat. no. sc-509, 1:500), Bcl-xL (cat. no. sc-8392, 1:500) and $\beta$-actin (cat. no. sc-47778, 1:1,000) from Santa Cruz Biotechnology, Inc.; p-Akt (cat. no. 9271, 1:1,000) and caspase-3 (cat. no. 9662, 1:500) from Cell Signaling Technology, Inc. (Danvers, MA, USA). All other reagents were commercially available in China.

Cell lines and treatment. The RMG-I-H cell line, highly expressing the FUT1 gene and Lewis(y) antigen, was established by transfecting the pcDNA3.1(-)-HFUT-H expression vector (containing the FUT1 gene) into RMG-I cells (a human ovarian clear cell carcinoma cell line, donated by Professor Iwamori Masao of Tokyo University, Tokyo, Japan) (6). The SKOV3, A2780 and COC1 cells, and their cognate cisplatinresistant cells SKOV3/DDP, A2780/DDP, COC1/DDP were obtained from the American Tissue Culture Collection (ATCC, Manassas, VA, USA).

The cells were maintained in DMEM supplemented with $10 \%$ fetal bovine serum at $37^{\circ} \mathrm{C}$ in a humidified $5 \% \mathrm{CO}_{2}$ atmosphere. Cells in the exponential growth phase were used in the subsequent experiments. In the case of carboplatin treatment, carboplatin was added to the culture medium at $60 \mathrm{mg} / \mathrm{l}$ and the cells were incubated for $48 \mathrm{~h}$. For the inhibition assay, the final concentration of Lewis(y) antibody was $20 \mu \mathrm{g} / \mathrm{ml}$. The duration of treatment was $1 \mathrm{~h}$.

Immunohistochemistry. For SP immunohistochemistry, the following protocol was used. Tissues were fixed in $4 \%$ formaldehyde and embedded in paraffin. A colon cancer sample served as a positive control for Lewis(y) antigen. The group treated with phosphate-buffered saline (PBS) instead of primary antibody was used as a negative control. The working concentration of the primary antibody against Lewis(y) was 1:160. The empirical procedure was performed based on the kit instructions [streptavidin-biotin-peroxidase (SP) test kit].

Immunocytochemistry. The cells cultured in chamber slides were fixed with $4 \%$ of paraformaldehyde, then stained according to the SP test kit instructions. The primary antibody, mouse anti-human Lewis(y) antibody, was used at a 1:100 dilution. Lewis(y) immunostaining was performed using an avidin-biotin peroxidase complex kit and then slides were photographed. The presence of brownish-yellow granules in the cytoplasm and cell membrane were considered as a positive result.

Confocal laser scanning microscopy. The protocols strictly followed the instructions of the reagent suppliers. The ANXA4 antibody and Lewis(y) antibody were simultaneously added to monolayered cell slides prepared from the RMG-I-H cells. 
To these, the following secondary antibodies were applied: Fluorescein isothiocyanate green fluorescence-labeled mouse IgM fluorescence (cat. no. sc-2859; 1:8) and tetramethylrhodamine red fluorescence-labeled rabbit IgG (cat. no. sc-2492; 1:50) (both from Santa Cruz Biotechnology, Inc.). Cell nuclei were stained with 4',6-diamidino-2-phenylindole. For negative controls, PBS replaced the primary antibodies. Double-labeled immunofluorescence samples were viewed under a fluorescence confocal microscope (C1-SI; Nikon, Tokyo, Japan).

Western blot analysis. Briefly, after the various treatments, the cells were washed twice with ice-cold PBS, scraped in lysis buffer [50 mM Tris- $\mathrm{HCl}$ (pH 7.4), $150 \mathrm{mM} \mathrm{NaCl}, 0.5 \% \mathrm{NP}-40$, $100 \mathrm{mM} \mathrm{NaF}, 200 \mu \mathrm{M} \mathrm{Na}_{3} \mathrm{VO}_{4}$ and $10 \mu \mathrm{g} / \mathrm{ml}$ each aprotinin, leupeptin, phenylmethanesulfonyl fluoride and pepstatin], and incubated for $20 \mathrm{~min}$ at $4^{\circ} \mathrm{C}$ while rocking. Lysates were cleared by centrifugation $\left(15 \mathrm{~min}\right.$ at $\left.13,000 \mathrm{x} \mathrm{g}, 4^{\circ} \mathrm{C}\right)$. For western blot analysis, $50 \mu \mathrm{g}$ of total protein were resolved with 10\% SDS-PAGE and transferred onto poly (vinylidene difluoride) membranes. The membranes were blocked with Tris-buffered saline Tween $[25 \mathrm{mM}$ Tris-HCl, $150 \mathrm{mM} \mathrm{NaCl}$ (pH 7.5), and $0.1 \%$ Tween-20] containing 5\% non-fat milk and incubated overnight at $4{ }^{\circ} \mathrm{C}$ with primary antibody in TBST/1\% non-fat milk. The blots were washed in TTBS and incubated with the appropriate horseradish peroxidase-linked $\mathrm{IgG}$ at room temperature for $1 \mathrm{~h}$, and immunoreactive proteins were visualized using an ECL detection system. The protein bands were visualized using the Molecular Imager system GDS8000b (UVP, Inc., Upland, CA, USA). Total protein levels were normalized to $\beta$-actin expression on the same membrane, and the bands were quantified using ImageJ software v1.8.0 (National Institutes of Health, Bethesda, MD, USA).

Co-immunoprecipitation. The cold PBS washed monolayer cells were lysed with $200 \mu 1$ lysis buffer as described above. The protein content was measured using the protein assay BCA kit (Beyotime Biotechnology, Jiangsu, China). Following protein determination, cell lysate containing $500 \mu \mathrm{g}$ protein was incubated with $5 \mu \mathrm{g}$ of one of the following antibodies, and incubated at $4^{\circ} \mathrm{C}$ overnight. Protein $\mathrm{G}$ plus-agarose was added and the samples were incubated at $4^{\circ} \mathrm{C}$ for $3 \mathrm{~h}$ for immunoprecipitation. ANXA4 was subjected to 10\% SDS-PAGE, then transferred onto a poly (vinylidene difluoride) membrane and treated with 1:500 diluted anti-Lewis(y) and anti-ANXA4 sera in Tris-buffered saline with 5\% non-fat milk, followed by HRP-labeled secondary antibody at a 1:1,000 dilution. Finally, the proteins were visualized with ECL reagent (ECL Prime Western Blotting Detection Reagent, Amersham, Pittsburgh, PA, USA). The densitometric analysis of the protein bands was performed using Image J software v1.8.0 (National Institutes of Health, Bethesda, MD, USA).

Reverse transcription-quantitative PCR (RT- $q P C R)$. Total RNA was extracted from the ovarian cancer cells using TRIzol reagent (Invitrogen, Shanghai, China). Complementary DNA (cDNA) was synthesized using reverse transcriptase from a Perfect Real-Time PrimeScript ${ }^{\mathrm{TM}}$ RT Reagent kit (Takara Bio, Inc.). The reaction conditions were as follows: $37^{\circ} \mathrm{C}$ for $15 \mathrm{~min}, 85^{\circ} \mathrm{C}$ for $5 \mathrm{sec}, 4^{\circ} \mathrm{C}$ for $5 \mathrm{~min}$. Real-time PCR and Ct value analysis were performed with a Roche LightCycler system. The primers
Table I. Primer sequences used for RT-qPCR.

\begin{tabular}{ll} 
Gene name & \multicolumn{1}{c}{ Primer sequence (5'-3') } \\
\hline FUT1 & F: AGGTATAAACACACCCTCTGTGCTT \\
& R: GAGTTCAGGGACAGACAGTGGTT \\
ANXA4 & F: AGCCTACAAGAGCACCATCG \\
& R: GACAGAGACACCAGCACTCG \\
BIK & F: GGTTCTTGGCATGACTGA \\
& R: GGCCAATGCGTCACT \\
TM4SF4 & F: CTTCCACGACGGGGATTAT \\
& R: ATTGTAGTCGTCATGCTGTAGAGTC \\
PHLDAl & F: TCATCCTTACTCTCACCCGC \\
& R: CTGGAGTTGGTACGGGTGAG \\
GAPDH & F: CCTTCATTGACCTCCACTAC \\
& R: GTTGTCATACTTCTCATGGTTC
\end{tabular}

$\mathrm{F}$, forward primer; R, reverse primer. FUT1, fucosyltransferase 1; ANXA4, Annexin A4; BIK, BCL2 interacting killer; TM4SF4, transmembrane $4 \mathrm{Lsix}$ family member 4; $P H L D A 1$, pleckstrin homology-like domain family A member 1; GAPDH, glyceraldehyde 3-phosphate dehydrogenase.

of target genes were commercially synthesized (Table I). The real-time PCR reaction conditions were denature at $95^{\circ} \mathrm{C}$ for $30 \mathrm{sec}, 40 \mathrm{cycles}$ of $95^{\circ} \mathrm{C}$ for $5 \mathrm{sec}$ and $60^{\circ} \mathrm{C}$ for $30 \mathrm{sec}$ in a $20-\mu \mathrm{l}$ reaction mixture containing $\mathrm{SYBR}^{\circledR}$ Premix Ex Taq ${ }^{\mathrm{TM}}$ (2X) $10 \mu \mathrm{l}$, PCR Forward Primer (10 $\mu \mathrm{mol} / \mathrm{l}) 0.4 \mu \mathrm{l}$, PCR Reverse Primer (10 $\mu \mathrm{mol} / \mathrm{l}) 0.4 \mu \mathrm{l}$, cDNA $2 \mu \mathrm{l}, \mathrm{dH}_{2} \mathrm{O} 7.2 \mu \mathrm{l}$. GAPDH was used as an endogenous control. Relative quantification was performed according to the $\Delta \Delta \mathrm{Cq}$ method, and results were expressed in the linear form using the formula $2^{-\Delta \Delta \mathrm{Cq}}(14)$. Results were considered significant when at least a 2 -fold difference in expression levels was detected.

Functional and pathway enrichment analysis. Using human whole genome oligonucleotide microarrays (Agilent whole human genome oligo microarray; Agilent Technologies, Santa Clara, CA, USA), gene expression profile alteration in response to Lewis(y) was investigated and 390 DEGs were identified and validated in the epithelial ovarian cell lines (RMG-I and COC1) compared with their sublines (RMG-I-H and $\mathrm{COC} 1 / \mathrm{DDP})$ with greater resistance to chemotherapy, in which 229 genes were upregulated and 161 genes were downregulated (15). Gene ontology (GO) and Kyoto Encyclopedia of Genes and Genomes (KEGG) pathway enrichment analysis were performed for the identified DEGs using the Database for Annotation, Visualization and Integrated Discovery (DAVID) database. A value of $\mathrm{P}<0.05$ was set as the cut-off criterion.

Protein-protein interaction (PPI) network construction and module selection. The functional interactions between proteins can provide context for the molecular mechanism of cellular processing. In this study, a PPI network of DEGs was constructed using the Search Tool for the Retrieval of Interacting Genes (STRING, http://string.embl.de/) database and subsequently was visualized using Cytoscape. A confidence score $\geq 0.4$ was 
Table II. Expression of Lewis(y) antigen in the different group.

\begin{tabular}{|c|c|c|c|c|c|c|c|}
\hline \multirow[b]{2}{*}{ Group } & \multirow[b]{2}{*}{$\mathrm{N}$} & \multicolumn{4}{|c|}{ Lewis(y) antigen } & \multirow[b]{2}{*}{ Positive cases } & \multirow[b]{2}{*}{ Positive rate $(\%)$} \\
\hline & & - & + & ++ & +++ & & \\
\hline Resistant group & 37 & 3 & 8 & 18 & 8 & 34 & $91.89^{\mathrm{a}}$ \\
\hline Sensitive group & 55 & 21 & 16 & 18 & 0 & 34 & 61.82 \\
\hline
\end{tabular}

${ }^{\mathrm{a} C}$ Compared with the control group, $\mathrm{P}<0.05$.

set as the cut-off criterion. Subsequently, Molecular Complex Detection (MCODE) was performed to screen the modules of the PPI network with a degree cut-off of 2 , a node score cut-off of 0.2 , a k-core of 2 , and a max. depth of 100 . Finally, the functional enrichment analysis of genes in each module was performed using the DAVID database.

Statistical analysis. SPSS 17.0 statistical software (IBM, Armonk, NY,USA) was applied for statistical analysis. Statistical methods were varied based on the data type. Quantitative data are presented as the means $\pm \mathrm{SD}$. As for the analysis of the data from RT-qPCR, data are expressed as the means \pm SEM. Positive ratio rates were evaluated using the Chi-square $\left(\chi^{2}\right)$ test. A Student's t-test was employed for comparisons between 2 groups and one-way ANOVA with the LSD or Bonferroni post hoc test was used for comparisons between $>2$ groups. A P-value $<0.05$ was considered to indicate a statistically significant difference.

\section{Results}

Expression of Lewis(y) antigen and the FUT1 gene in ovarian cancer tissues and cells. Our results revealed that in the 92 samples of ovarian cancer tissues, Lewis(y) antigen was mainly expressed on the cell membrane and to a lesser extent in the cytoplasm (Fig. 1A). The positive rate of Lewis(y) antigen in chemoresistant ovarian cancer tissues was $91.89 \%$, which was significantly higher than that in the sensitive group $(61.82 \%, \mathrm{P}<0.05)$ (Table II). The expression intensity of Lewis(y) antigen in the resistant group was also significantly higher than that in the sensitive group. In the resistant group, 8 of the 34 positive cases exhibited a strong positive expression, while in the sensitive group, there were no strongly positive cases among the 34 positive cases (Table II).

In previous studies, we introduced the FUT1 gene into human ovarian carcinoma-derived RMG-I cells through gene transfection and established a cell model overexpressing the FUT1 gene and Lewis(y) antigen. Further experiments demonstrated that the FUT1-transfected cells exhibited a reduced sensitivity to common chemotherapeutic drugs, such as carboplatin, 5-FU and paclitaxel (6-8). In this study, to further examine whether the expression of Lewis(y) antigen was increased when the cells became resistant to chemotherapy, the expression of Lewis(y) antigen and FUT1 in ovarian cancer cells was detected by immunocytochemical staining and RT-qPCR, respectively. The results revealed that after the cells were challenged by chemotherapeutic drugs, the expression of Lewis(y) antigen in the cisplatin-resistant cell lines (SKOV3/DDP, COC1/DDP and A2780/DDP) was significantly higher than the expression in their parental non-resistant cell lines (SKOV3, COC1 and A2780); the antigen was also widely distributed on the cell membrane (Fig. 1B). The results of RT-qPCR confirmed that the mRNA expression level of FUT1 in the SKOV3/DDP cells increased by 3.17-fold in comparison to that in the SKOV3 cells, the mRNA expression level of FUT1 in the COC1/DDP cells increased by 2.42-fold in comparison to that in the COC1 cells, and the mRNA expression level of FUT1 in the A2780/DDP cells increased by 2.84 -fold in comparison to that in the A2780 cells (all $\mathrm{P}<0.05$ ) (Fig. 1C).

Functional and pathway enrichment analysis. GO enrichment analysis revealed that the DEGs were mainly involved in the transmembrane receptor protein tyrosine kinase signaling pathway, the positive regulation of cell proliferation, the integrin-mediated signaling pathway, extracellular matrix organization and angiogenesis (Fig. 2 and Table III).

KEGG pathway analysis of the DEGs suggested that these genes were significantly enriched in antigen processing and presentation, fructose and mannose metabolism, and central carbon metabolism in cancer (Fig. 3 and Table IV).

PPI network construction and module selection. We also constructed a PPI network to examine the mechanisms through which these genes interact with each other, as well as to identify the central node of the PPI network. The PPI network of the DEGs consisted of 323 nodes and 287 edges. A significant module was obtained from the PPI network of DEGs using MCODE, including 18 nodes and 45 edges (Fig. 4A and B). Functional enrichment analysis revealed that genes in this module were mainly associated with the regulation of the apoptotic process, the positive regulation of release of the cytochrome $c$ from the mitochondria, and protection from natural killer cell-mediated cytotoxicity (Fig. 4C).

Validation of the DEGs. Four DEGs, ANXA4, BCL2 interacting killer $(B I K)$, transmembrane $4 \mathrm{~L}$ six family member 4 (TM4SF4) and pleckstrin homology like domain family A member 1 (PHLDAl), were selected to validate the results of gene chip analysis. The results of RT-qPCR revealed that the mRNA levels of the 4 genes were significantly increased in the Lewis(y) highly-expressing chemoresistant ovarian cancer cells (RMG-I-H and COC1/DDP cells), exhibiting 2.82- (ANXA4), 4,21- (BIK), 9.04- (TM4SF4) and 3.38-fold (PHLDA1) increases over the expression levels in the non-resistant cells (RMG-I and COC1 cells) $(\mathrm{P}<0.01)$ (Fig. 5). This was consistent with the results of gene chip analysis. These results suggest that Lewis(y) causes 

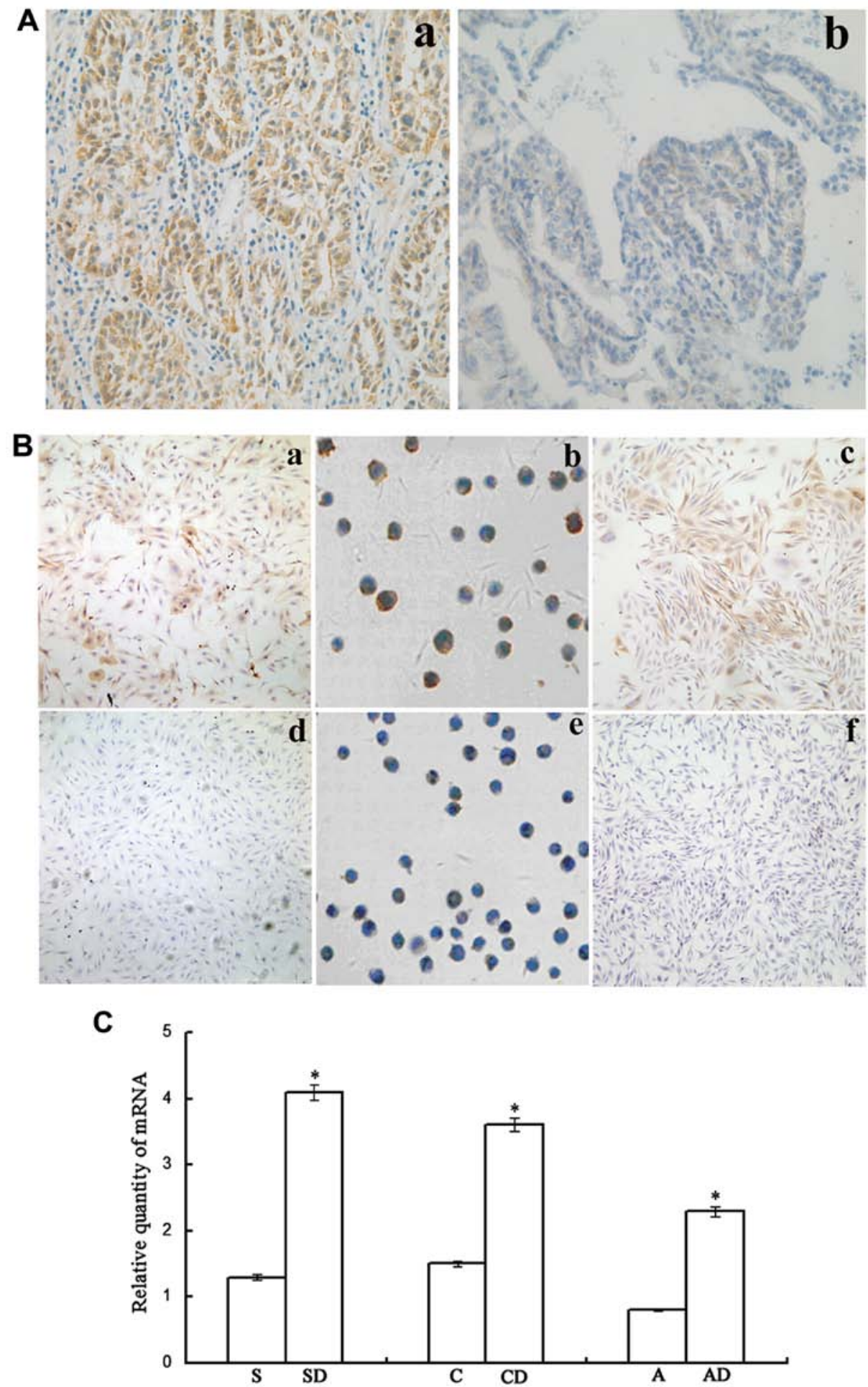

Figure 1. Association between Lewis(y) antigen and chemotherapy resistance in ovarian cancer.(A) Immunohistochemical detection of the expression of Lewis(y) antigen in ovarian cancer tissues (magnification, x200). (a) Chemotherapy-resistant group; (b) chemotherapy-sensitive group. (B) Immunocytochemical detection of Lewis(y) antigen expression in 3 groups of ovarian cancer cells with varying degrees of malignancy. (a-c) Chemotherapy-resistant cells (SKOV3/DDP, COC1/DDP and A2780/DDP cells); (d-f) chemotherapy-sensitive cells (SKOV3, COC1 and A2780 cells). (C) RT-qPCR detection of FUT1 expression in 3 groups of ovarian cancer cells with varying degrees of malignancy. " $\mathrm{P}<0.05$, SKOV3/DDP compared to SKOV3 cells, COC1/DDP compared to COC1 cells, and A2780/DDP compared to A2780 cells. Bars are labeled as follows: S, SKOV3 cells; SD, SKOV3/DDP cells; C, COC1 cells; CD, COC1/DDP cells; A, A2780 cells; AD, A2780/DDP cells. FUT1, fucosyltransferase 1.

cancer chemotherapeutic resistance may be due to the inhibition of apoptosis.

Co-expression of ANXA4 and Lewis(y) antigen in ovarian cancer cells. As ANXA4 is a membrane phospholipid-binding protein, we further investigated the protein expression of ANXA4 in ovarian cancer cells and the presence of Lewis(y) antigen in this protein. The results of western blot analysis revealed that the protein expression of ANXA4 was significantly increased in the ovarian cancer chemotherapy-resistant 


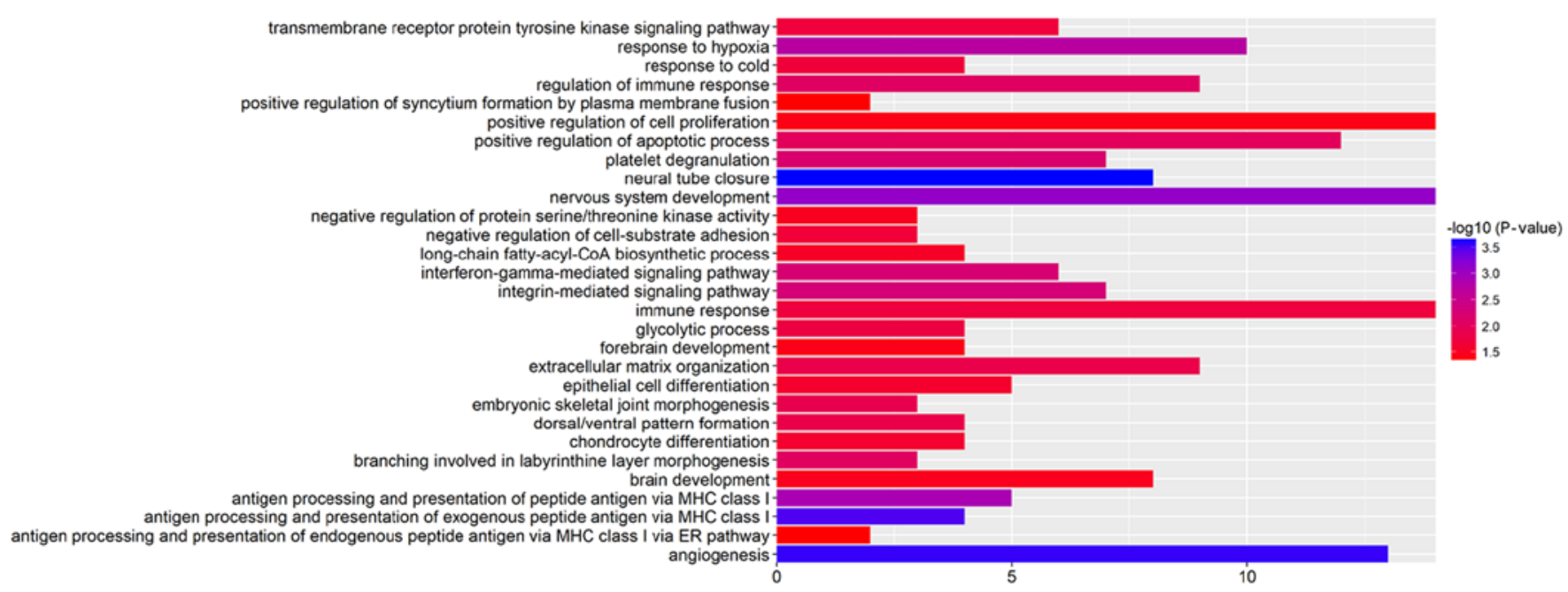

Figure 2. Significantly enriched GO terms of DEGs in response to Lewis(y) antigen in ovarian cancer cells based on their functions. GO, Gene Ontology; DEGs, differentially expressed genes.

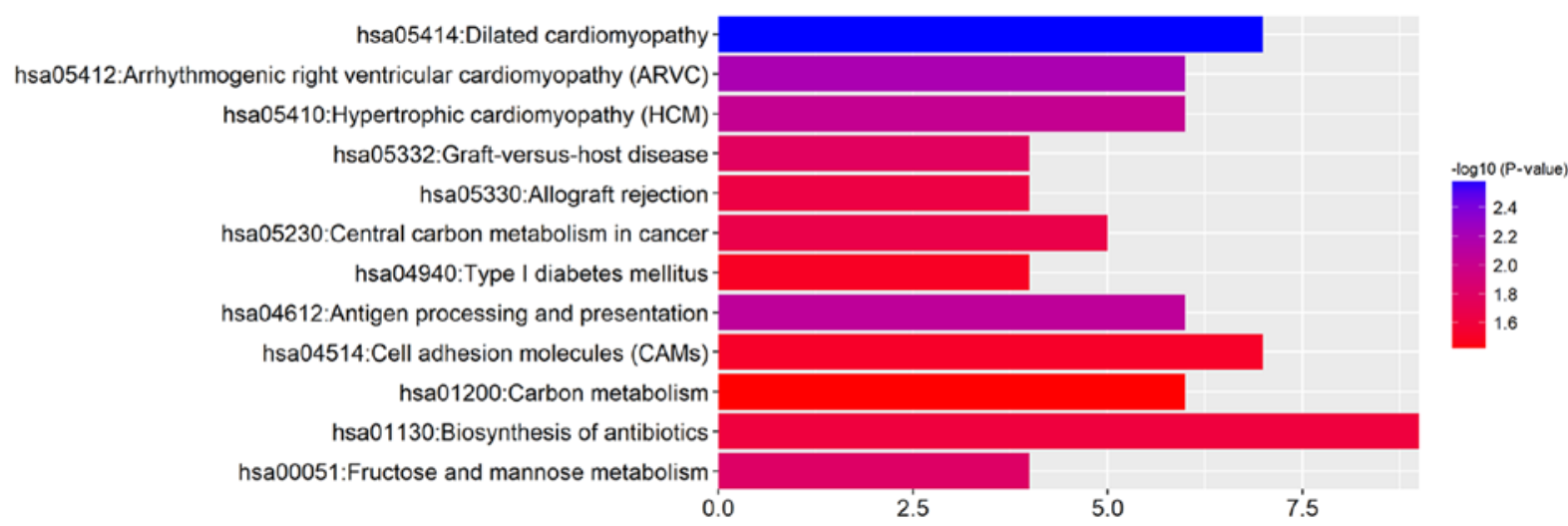

Figure 3. Signaling pathway enrichment analysis of DEGs in response to Lewis(y) antigen in ovarian cancer cells. DEGs, differentially expressed genes.

cells (RMG-I-H and COC1/DDP cells) expressing high levels of Lewis(y), with an expression level increase of 2.72 -fold and 2.24-fold over the level in RMG-I cells and COC1 cells, respectively, exhibiting the same trend as the mRNA expression (Fig. 6A).

Co-immunoprecipitation and laser confocal microscopy were used to determine whether there was a Lewis(y) structure in the ANXA4 protein. The results revealed that ANXA4 co-immunoprecipitated with Lewis(y) antigen corresponding to the $36 \mathrm{kDa}$ band (Fig. 6B). Dual-labeling immunofluorescence revealed red immunofluorescence, indicating that the Lewis(y) antigen was mainly distributed in the membrane, while green immunofluorescence indicated ANXA4 was also mainly distributed in the membrane, although it could also be observed in the cytoplasm. Moreover, red and green immunofluorescence were overlaid in the membrane, suggesting the co-localization of Lewis(y) antigen and ANXA4 (Fig. 6C).

Differences in apoptosis-related protein expression in ovarian cancer cells. We further examined the expression of apoptosis-related proteins, such as Bcl-2 in ovarian cancer cells. Following carboplatin treatment, the RMG-I-H cells exhibited an upregulated expression of the pro-apoptotic protein, Bax, by $57 \%$, and the phosphorylation of the anti-apoptotic protein, Bad, markedly increased by $197 \%$, compared with the pretreatment protein levels (Fig. 7A) (Bad itself is a pro-apoptotic protein that becomes anti-apoptotic upon phosphorylation by Akt.) The expression of other anti-apoptotic proteins, such as $\mathrm{Bcl}-2$ and $\mathrm{Bcl}-\mathrm{xL}$, was not noticeably altered following carboplatin treatment. Before treatment, the Bax expression level in the RMG-I cells was higher than that in the RMG-I-H cells by approximately 3.95 -fold. In addition, the posttreatment expression of Bax in the RMG-I cells increased by approximately 6.02 -fold greater than the increase exhibited by the RMG-I-H cells. The phosphorylation levels of Bad, and the levels of Bcl-2 and Bcl-xL in the RMG-I-H cells significantly increased by 4.64-, 1.91- and 2.07-fold as much as in the RMG-I cells, respectively. Following carboplatin treatment, the phosphorylation level of Bad in the RMG-I-H cells was approximately 7.78-fold higher than that in the RMG-I cells. Following carboplatin treatment, there was no evident change in the levels of phosphorylated Bad, and the levels of Bcl-2, or Bcl-xL in the RMG-I cells.

We also examined the expression of caspase-3, and found that, in the absence of carboplatin, caspase- 3 existed in its inactive, pro-enzyme form in the RMG-I and RMG-I-H cells, 
Table III. Top 12 significantly differentially expressed genes determined by enriched analysis in response to Lewis(y) antigen in ovarian cancer cells.

\begin{tabular}{llrl}
\hline Term & \multicolumn{1}{c}{ Description } & Count & P-value \\
\hline GO:0001525 & Angiogenesis & 13 & $2.72 \mathrm{E}-04$ \\
GO:0007399 & Nervous system development & 14 & $7.89 \mathrm{E}-04$ \\
GO:0001666 & Response to hypoxia & 10 & 0.001899323 \\
GO:0007229 & Integrin-mediated signaling pathway & 7 & 0.005265788 \\
GO:0060333 & Interferon-gamma-mediated signaling pathway & 6 & 0.005686303 \\
GO:0050776 & Regulation of immune response & 9 & 0.008269841 \\
GO:0043065 & Positive regulation of apoptotic process & 12 & 0.009485865 \\
GO:0030198 & Extracellular matrix organization & 9 & 0.014169248 \\
GO:0007169 & Transmembrane receptor protein tyrosine kinase signaling pathway & 6 & 0.019359692 \\
GO:0002062 & Chondrocyte differentiation & 4 & 0.024541795 \\
GO:0030855 & Epithelial cell differentiation & 5 & 0.026205904 \\
GO:0008284 & Positive regulation of cell proliferation & 14 & 0.039256986 \\
\hline
\end{tabular}

Table IV. Signaling pathway enrichment analysis of differentially expressed genes in response to Lewis(y) antigen in ovarian cancer cells.

\begin{tabular}{lllr}
\hline Term & \multicolumn{1}{c}{ Description } & Count & P-value \\
\hline hsa05414 & Dilated cardiomyopathy & 7 & 0.002639 \\
hsa05412 & Arrhythmogenic right ventricular cardiomyopathy (ARVC) & 6 & 0.006362 \\
hsa04612 & Antigen processing and presentation & 6 & 0.008456 \\
hsa05410 & Hypertrophic cardiomyopathy (HCM) & 6 & 0.009414 \\
hsa00051 & Fructose and mannose metabolism & 4 & 0.015682 \\
hsa05332 & Graft-versus-host disease & 4 & 0.017045 \\
hsa05230 & Central carbon metabolism in cancer & 5 & 0.021516 \\
hsa05330 & Allograft rejection & 4 & 0.023141 \\
hsa01130 & Biosynthesis of antibiotics & 9 & 0.024633 \\
hsa04514 & Cell adhesion molecules (CAMs) & 7 & 0.030688 \\
hsa04940 & Type I diabetes mellitus & 4 & 0.032215 \\
hsa01200 & Carbon metabolism & 6 & 0.039745 \\
\hline
\end{tabular}

and thus no hydrolysis of caspase-3 was observed (Fig. 7B). The hydrolytic breakdown of caspase- 3 into a small fragment subunit is required for its activation. The caspase- 3 pro-enzyme level in the RMG-I cells was approximately 1.6-fold higher than that in the RMG-I-H cells. Treatment with carboplatin markedly increased the level of active caspase- 3 in the RMG-I cells to a level approximately 4.03 -fold as much as that in the RMG-I-H cells. However, the level of the caspase-3 pro-enzyme exhibited no apparent changes in response to carboplatin treatment in either cell line.

Effect of Lewis(y) antibody on the expression of apoptosis related proteins in ovarian cancer cells following carboplatin treatment. To further determine whether the Lewis(y) antigen is involved in the inhibitory effects on the apoptosis of ovarian cancer cells, we used Lewis(y) antibody to treat the cells before carboplatin treatment. The phosphorylation levels of Akt and Bad were decreased in both cell lines in response to Lewis(y) antibody pre-treatment, although this decrease was greater in the RMG-I-H cells, which overexpress Lewis(y) (Fig. 8). The active fragment of caspase- 3 was increased in both cell lines, but more predominantly in the RMG-I-H cells (Fig. 8). Highand low-level differences between the RMG-I and RMG-I-H cells with regard to the levels of phosphorylated Akt, phosphorylated Bad and caspase-3 fragments were also suppressed after Lewis(y) antibody pre-treatment.

\section{Discussion}

Lewis antigens are a group of carbohydrate antigens present on cell surface glycolipids or glycoproteins. They are also tumor-associated carbohydrate antigens that are abnormally highly expressed in malignant tumors derived from epithelial tissues, such as the breast, ovaries, colon and rectum. A high expression of the Lewis $(\mathrm{y})$ antigen has been shown to be associated with a poorer prognosis of cancer $(16,17)$. Our previous studies demonstrated that approximately $80 \%$ of ovarian cancer samples had an increased expression of Lewis(y). 
A

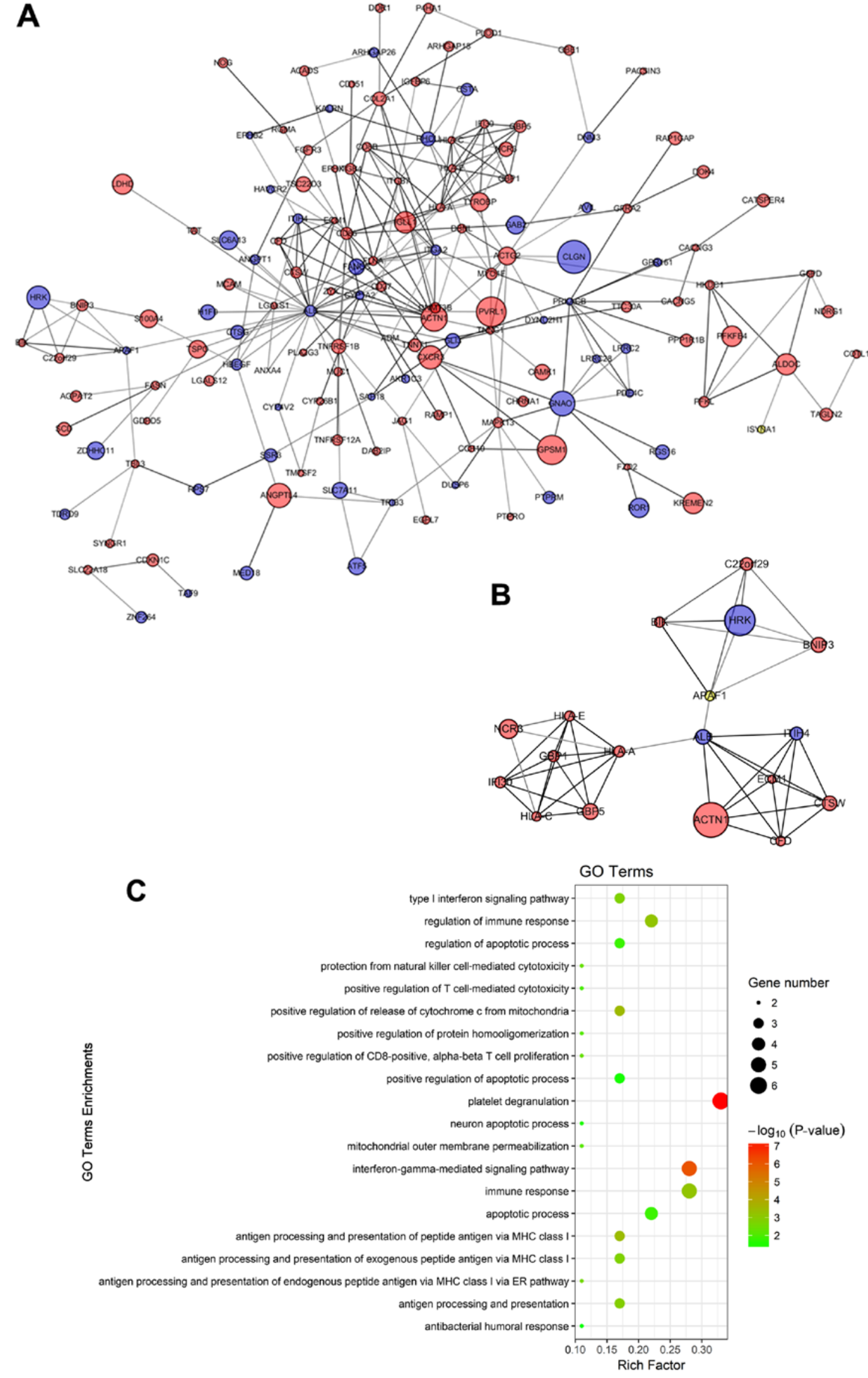

Figure 4. Protein-protein interaction network and a significant module. (A) Protein-protein interaction network of differentially expressed genes. (B) A significant module selected from the protein-protein interaction network. Red nodes indicate upregulated genes, while blue nodes indicate downregulated genes; the larger the node, the greater the difference in gene expression. The lines represent interaction associations between nodes. (C) Functional enrichment analysis of genes in the module. 

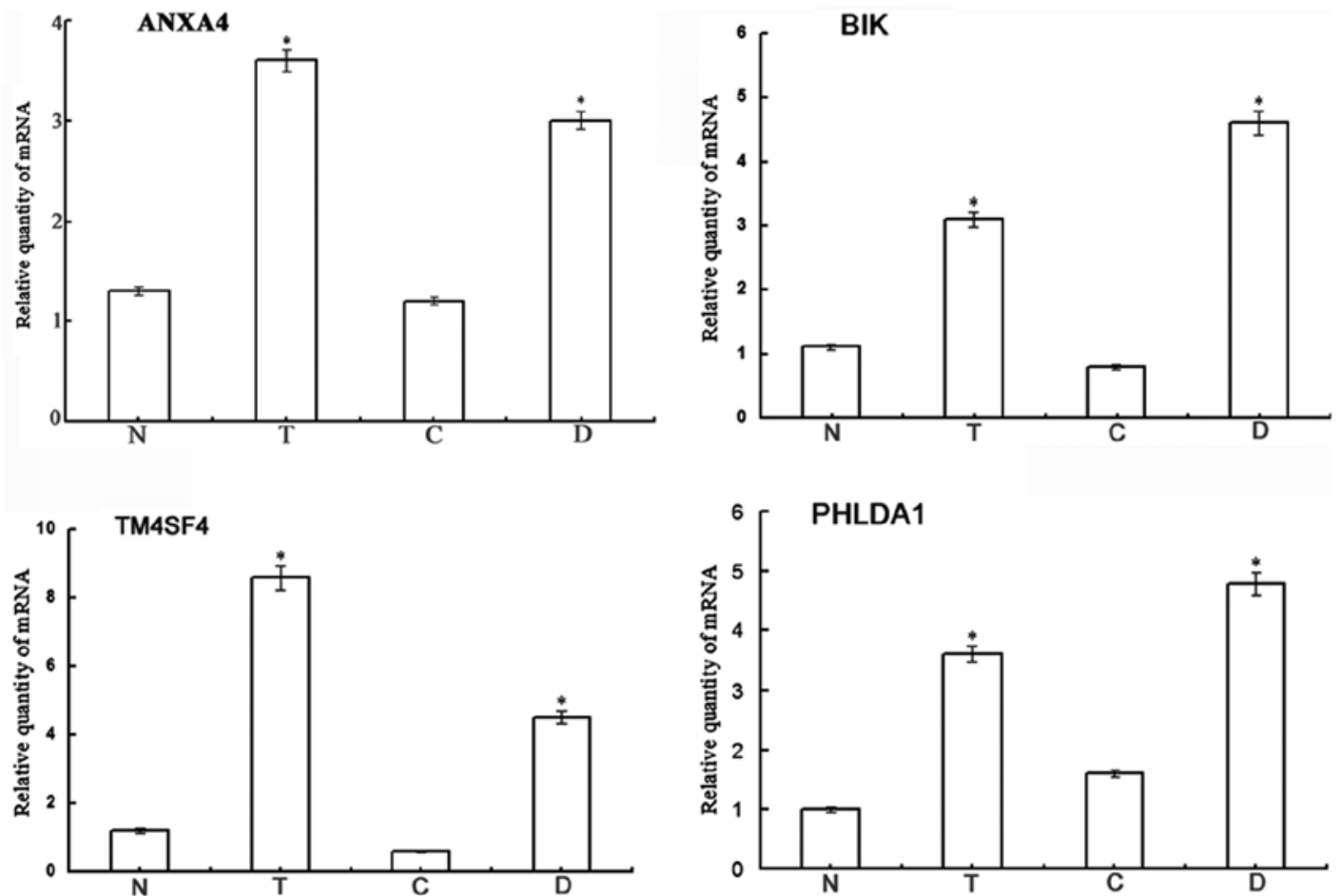

Figure 5. Validation of the differentially expressed genes. The results of RT-qPCR revealed that the mRNA expression levels of 4 selected genes (ANXA4,BIK, TM4SF4 and PHLDA1) exhibited obvious differences among the 4 ovarian cancer cell lines (RMG-I-H, RMG-I, COC1/DDP and COC1 cells). Bars are labeled as follows: N, RMG-I cells; T, RMG-I-H cells; C, COC1cells; D, COC1/DDP cells. "P<0.01, RMG-I-H compared to RMG-I cells, and COC1/DDP compared to COC1 cells. ANXA4, Annexin A4; BIK, BCL2 interacting killer; TM4SF4, transmembrane 4 L six family member 4; PHLDA1, pleckstrin homology-like domain family A member 1 .

This change in Lewis(y) expression affected the biological behaviors of ovarian cancer cells. Using an anti-Lewis(y) monoclonal antibody to block the antigen or $\alpha$-L-fucosidase to digest cell surface sugar chain antigens, we found that the proliferation and adhesion of ovarian cancer cells decreased, and their sensitivity to chemotherapy increased $(9,18-20)$. In the current study, the chemoresistance of ovarian cancer cells was induced and the expression of Lewis(y) was found to be significantly increased after the induction of chemoresistance. Combined with the clinical drug resistance observed in patients with ovarian cancer, this study suggests a significant association between Lewis(y) and drug resistance. In the chemoresistant group, the positive expression rate and expression intensity of Lewis(y) were both significantly higher than those in the sensitive group. Our previous study also found that Lewis(y)-associated drug resistance was not only closely related to the upregulation of certain drug resistant genes, including multidrug resistance protein $(M D R)-1$, multidrug resistance-associated protein $(M R P)-1, M R P-2$ and protein kinase $\mathrm{C}(P K C)-\alpha(21)$, but was also involved in the upregulation of some cell repair genes, such as topoisomerase (Topo)-I and Topo-II $\beta$ (22). Lewis(y) may thus be a novel target for reversing the chemoresistance of ovarian cancer cells. Moreover, the analysis of the molecular mechanisms responsible for the Lewis(y)-induced chemoresistance may provide theoretical support for improving the treatment efficacy of ovarian cancer.

In this study, we used a gene chip assay to analyze the gene expression profiles of a cisplatin-resistant ovarian cancer cell line with high expression of Lewis(y) antigen and its parental non-resistant cell line. There were 390 genes differentially expressed between the drug-resistant and the non-resistant cell lines. These DEGs were mainly associated with transmembrane receptor protein tyrosine kinase signaling pathway, the positive regulation of cell proliferation, the integrin-mediated signaling pathway, extracellular matrix organization, the regulation of apoptotic process, the positive regulation of release of cytochrome $c$ from mitochondria and antigen processing and presentation. We constructed an interaction network map and predicted a large number of interacting genes, which may prove to be helpful for understanding the mechanisms of the chemoresistance of ovarian cancer cells.

The majority of cell surface receptors are glycoproteins. Changes in the expression of glycosyltransferase can affect the sugar chain structure of cell surface receptors, which further affects the abundance and functions of these receptors (23). In our previous study, we found that Lewis(y), which is a bi-fucosyl-containing oligosaccharide chain, was a component of multiple membrane proteins, including epidermal growth factor receptor (EGFR), integrin $\alpha 5 \beta 1$, transforming growth factor beta receptor (TGF- $\beta R$ ) and HE4 (13,19,24-26). Furthermore, Lewis(y) expression increased with the increase in the malignant degree of ovarian cancer tissues or cells. In the present study, we found that ANXA4 was abnormally increased at both the mRNA and protein levels in drug-resistant ovarian cancer cells. ANXA4 is an important member of the membrane protein family, and we found that Lewis(y) antigen was also present in ANXA4 protein. Recent studies suggest that abnormally-high expression of ANXA4 occurs in many cancer types, including ovarian cancer; ANXA4 is 
A
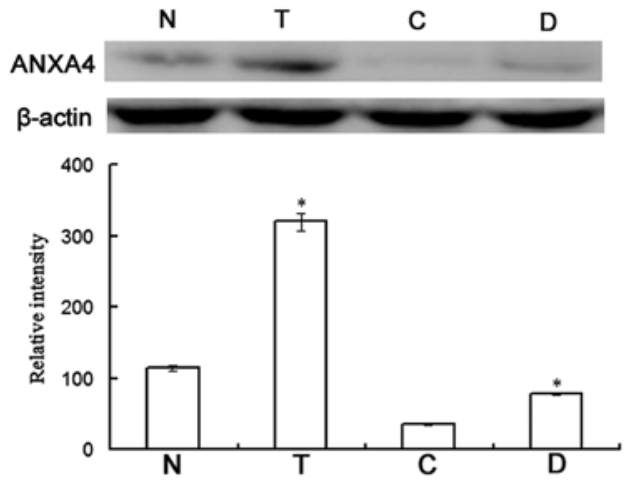

B

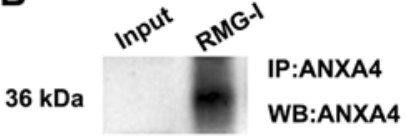

$36 \mathrm{kDa}$

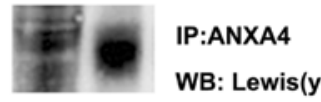

C
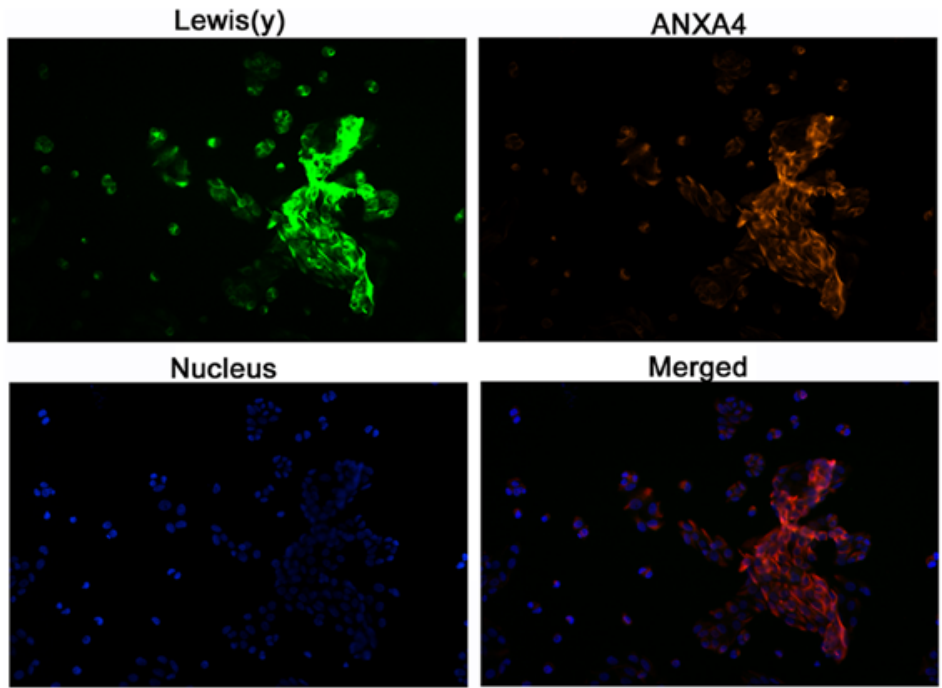

Figure 6 Co-expression of ANXA4 and Lewis(y) antigen in ovarian cancer cells. (A) Western blot analysis detection of ANXA4 protein expression in the 4 ovarian cancer cell lines (RMG-I-H, RMG-I, COC1/DDP and COC1 cells). Lanes and bars are labeled as follows: N, RMG-I cells; T, RMG-I-H cells; C, COC1cells; D, COC1/DDP cells. *P<0.01, RMG-I-H compared to RMG-I cells, and COC1/DDP compared to COC1 cells. (B) Immunoprecipitation indicated that Lewis(y) was expressed on the ANXA4 surface in ovarian cancer cells. (C) Double-labeling immunofluorescence showed the co-localization of ANXA4 and Lewis(y) in RMG-I cells. ANXA4, Annexin A4.
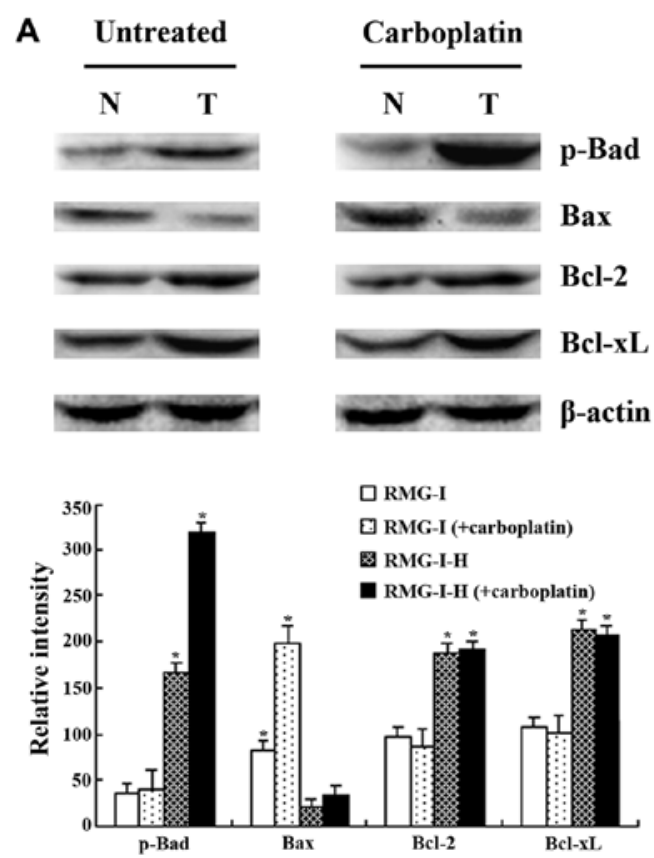

B
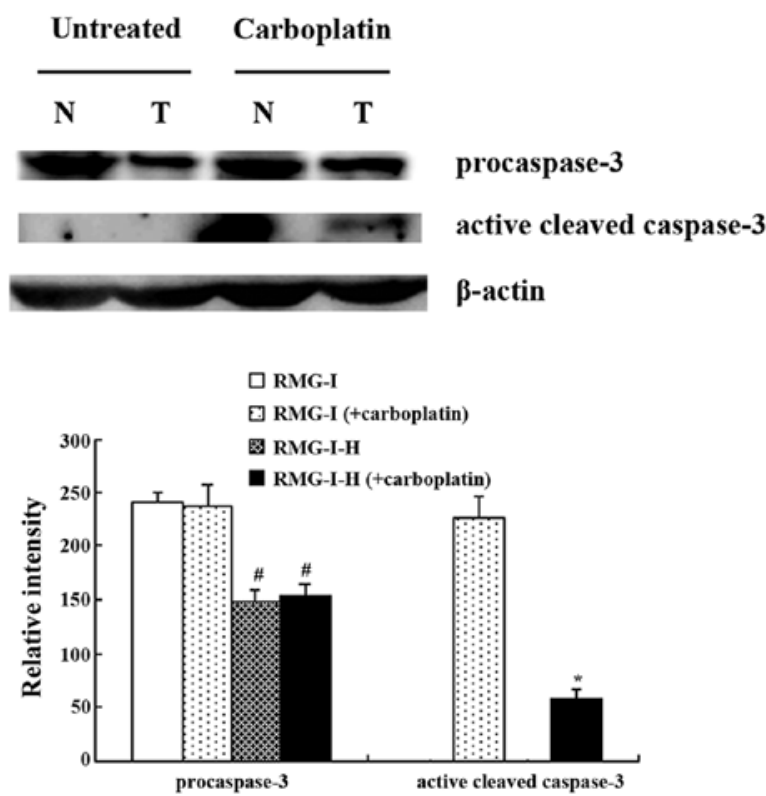

Figure 7. Differences in apoptosis-related protein expression in ovarian cancer cells. Western blot analysis detection of differences in (A) Bcl-2 family protein and (B) caspase-3 expression in ovarian cancer cells (RMG-I and RMG-I-H cells) following treatment with carboplatin. In the case of carboplatin treatment, carboplatin was added to the culture medium at $60 \mathrm{mg} / \mathrm{l}$ and the cells were incubated for $48 \mathrm{~h}$. Lanes are labeled as follows: N, RMG-I cells; T, RMG-I-H cells; p-Bad, phosphorylated Bad; cleaved caspase-3, active cleaved caspase-3. ${ }^{\prime} \mathrm{P}<0.05$ compared to RMG-I cells; " $\mathrm{P}<0.01$ compared to RMG-I cells. 
A

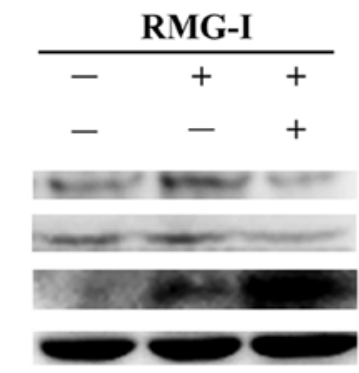

RMG-I-H

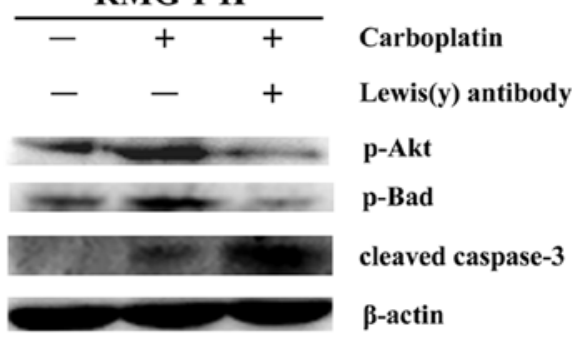

B

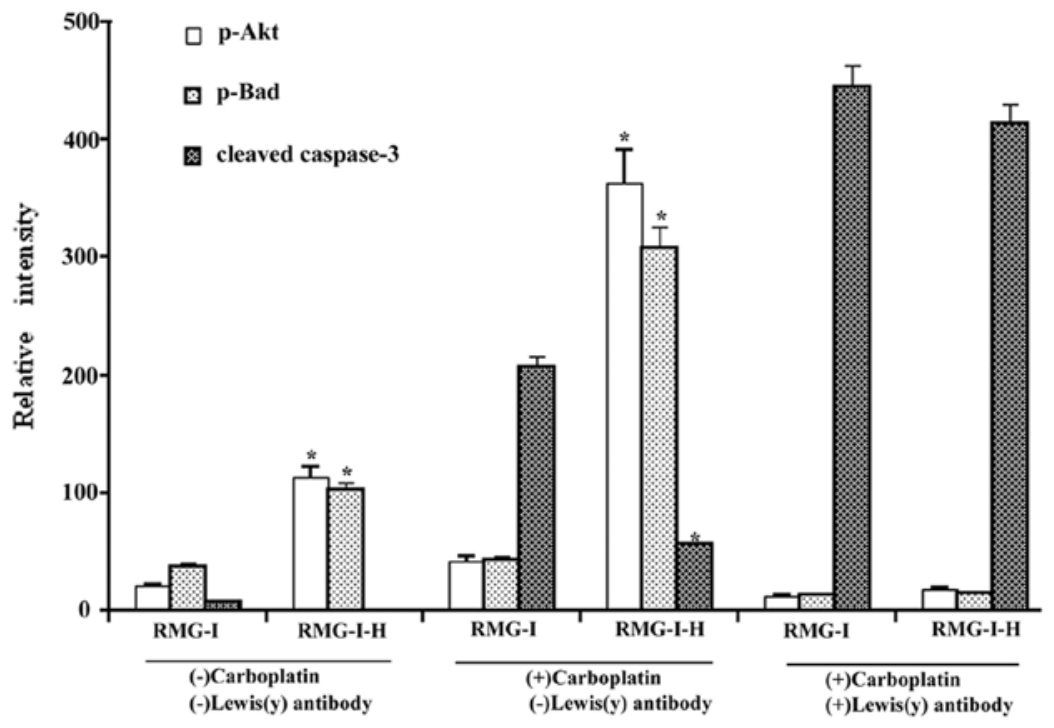

Figure 8. Lewis(y) antigen modification is involved in the inhibitory effect on apoptosis in ovarian cancer cells. Effect of Lewis(y) antibody on the expression of p-Akt, p-Bad and cleaved caspase-3 in cells following carboplatin treatment. (A) Representative western blots of phosphorylated Akt, phosphorylated Bad and active cleaved caspase- 3 in cell lines. (B) Densitometric quantification of protein expression of a $(n=3)$. In the case of carboplatin treatment, carboplatin was added to the culture medium at $60 \mathrm{mg} / \mathrm{l}$ and the cells were incubated for $48 \mathrm{~h}$. For the inhibition assay, the final concentration of Lewis(y) antibody was $20 \mu \mathrm{g} / \mathrm{ml}$. The duration of treatment was $1 \mathrm{~h}$. p-Akt, phosphorylated Akt; p-Bad, phosphorylated Bad; cleaved caspase-3, active cleaved caspase-3. ${ }^{*} \mathrm{P}<0.01$ compared to RMG-I cells.

also associated with the proliferation, apoptosis, metastasis, chemoresistance, and with other biological behaviors of tumor cells $(27,28)$. A previous study by Choi et al (29) indicated that ANXA4 was overexpressed in chemoresistant ovarian serous carcinoma tissues compared with chemosensitive tissues, which was consistent with our study. Lin et al (30) found that ANXA4 promoted the proliferation of gastric cancer cells; during this process, Akt was activated by phosphorylation to regulate the PI3K/Akt signaling pathway, leading to cell proliferation and resistance to chemotherapy or other stress conditions. In the present study, after the RMG-I-H cells were treated with the anti-Lewis(y) antibody, the expression of phosphorylated Akt was significantly decreased.

The mechanisms of malignant tumor resistance to chemotherapeutic drugs are complex and poorly understood; all chemotherapeutic drugs exert their antitumor effects ultimately by inducing the apoptosis of tumor cells. If an apoptosis signaling pathway becomes defective or anti-apoptotic mechanisms are enhanced, this may result in drug resistance in tumor cells. The process of apoptosis is tightly controlled in eukaryotes and consists of two main pathways: The mitochondrial and death receptor pathways. Bcl-2 family proteins, including the pro-apoptotic proteins, Bax and $\mathrm{Bad}$, and the apoptosisinhibitory proteins $\mathrm{Bcl}-2$ and $\mathrm{Bcl}-\mathrm{xL}$, play vital roles in the mitochondrial pathway of apoptosis. In this study, we found that regardless of carboplatin treatment, the levels of phosphorylated Bad, Bcl-2 and Bcl-xL in the RMG-I-H cells [which highly express Lewis(y) antigen] were higher than those in the RMG-I cells. Following carboplatin treatment, Bax expression in the RMG-I cells and p-Bad levels in the RMG-I-H cells were markedly upregulated, whereas Bcl-2 and Bcl-xL expression remained unaltered in both cell lines, compared with expression levels before carboplatin treatment. These results suggest that Lewis(y) may be involved in controlling apoptosis by regulating the expression of $\mathrm{Bcl}-2$ family proteins. Using a breast cancer cell line, Kelly et al (31) reported that exposure to the anti-Lewis(y) monoclonal antibody markedly increased the sensitivity to $\gamma$-rays and led to an increased number of cancer cells undergoing apoptosis. Baldus et al (32) found that the expression of Lewis(y) increased in colon mucinous cancer cells, and simultaneously, the number of apoptotic cells declined. Taken together, these results demonstrate that Lewis(y) is closely related to apoptosis. A homodimer or a heterodimer can be formed between most members of the Bcl-2 family, and heterodimers formed between pro-apoptotic and anti-apoptotic members repress opponent function. Thus, the ratio of $\mathrm{Bcl}-2(\mathrm{Bcl}-\mathrm{xL}) / \mathrm{Bax}$ determines the survival of cells. If $\mathrm{Bax}$ is dominant, Bax homodimers enhance sensitivity of 
cells to apoptosis-inducing signals. If $\mathrm{Bcl}-2$ or $\mathrm{Bcl}-\mathrm{xL}$ is dominant, Bcl-2(Bcl-xL)/Bax heterodimers exhibit antiapoptotic functions, which inhibit the release of cytochrome $c$ from the mitochondria to the cytoplasm and prevent activation of specific caspase family pro-enzymes (including caspase-3 proenzyme) by cytochrome $c$. Caspase- 3 is the primary apoptosis-executing protein that functions by partially or completely hydrolyzing proteins essential to cell survival, such as DNA repair-associated proteins. An increase in caspase-3 activity (i.e., the hydrolysis of the proenzyme to small fragments) indicates that cellular apoptosis has been initiated. We found that both the caspase-3 pro-enzyme and active fragment were expressed at lower levels in the RMG-I-H cells than in the RMG-I cells, regardless of carboplatin treatment. Our results also suggested that the overexpression of Lewis(y) antigen induced an increased expression of the apoptosis-inhibitory proteins, Bcl-2 and Bcl-xL, and a decreased expression of the pro-apoptosic protein, Bax. Consequently, the ratio of Bcl-2(Bcl-xL)/Bax increased, which further inhibited the activation of caspase-3 pro-enzyme, decreased the availability of caspase- 3 active fragments, inhibited cell apoptosis, and promoted the development of tumor drug resistance.

Azuma et al $(33,34)$ found that the expression of both FUT4 and its products, [Lewis(x) and Lewis(y)], increased after an anti-Fas antibody was used to induce the apoptosis of a human $\mathrm{T}$ cell line (Jurkat), and a caspase-3 inhibitor suppressed the increase in Lewis(y) antigen. Iwamori et al (16) reported that the levels of fucosyl antigens [e.g., Lewis(b) and Lewis(x)] on the cell surface increased markedly when a human ovarian cancer cell line (KFr13TX) exhibited drug resistance to paclitaxel. In our previous study, we found that c-Jun/AP-1 expression was increased in highly malignant ovarian cancer tissues and cells, and its expression was positively associated with the mRNA expression of FUT1 and Lewis(y) antigen. We also found that the promoter region of FUT1 contained an AP-1 response element; c-Jun/AP-1 can specifically bind to the promoter of FUT1 to promote FUT1 transcription and Lewis(y) antigen expression (35). In the present study, we found that after highly-expressing Lewis(y) ovarian cancer cells were treated with carboplatin, the increases in the levels of phosphorylated AKT were more significant than those before treatment.

In conclusion, the expression of Lewis (y) antigen is elevated in chemoresistant ovarian cancer cells, and ovarian cancer cells with a high expression of Lewis(y) are more likely to develop resistance, thus forming a positive feedback loop, which promotes the progression of ovarian cancer. Based on existing research and our experimental results, we hypothesized that ovarian cancer cells activate their own anti-apoptotic mechanism, while the apoptotic program is activated under the control of apoptosis-inducing factors, which promote Lewis(y) antigen expression; the increased expression of Lewis(y) antigen then upregulates the relevant pathways and ultimately enhances the anti-apoptotic activity of ovarian cancer cells. The potential mechanisms are as follows: Lewis(y) is a component of many membrane proteins including ANXA4; its expression increases with increasing malignancy of ovarian cancer tissues or cells; the overexpression of Lewis(y) can then activate downstream signaling pathways to regulate the expression of multiple factors and mediate drug resistance of cancer cells; Lewis(y) can also affect the expression and activity of c-Jun/AP-1 to regulate the activity of $\alpha 1,2$-fucosyltransferase and the transcription of FUT1, which promotes the expression of Lewis $(y)$ via a positive feedback mechanism. These effects eventually promote the development and progression of ovarian cancer.

\section{Acknowledgements}

Not applicable.

\section{Funding}

This study was supported by grants from The National Natural Science Foundation of China (nos. 81172491, 81101527, 81472437 and 81672590), and Outstanding Scientific Fund of Shengjing Hospital (no. 201303).

\section{Availability of data and materials}

All data generated or analyzed during this study are included in this published article.

\section{Authors' contributions}

JL carried out most parts of the experiment; MZ, YQ and HW participated in the experiments; BL participated in the conception and design of the study; ML and QL performed the statistical analysis. All authors have read and approved the final manuscript.

\section{Ethics approval and consent to participate}

Samples were fully encoded to protect patient confidentially and thus patient consent was waived by the Ethics Committee. The study and its protocols were approved by the Research Ethics committees of Shengjing Hospital Affiliated with China Medical University (2013PS66K).

\section{Patient consent for publication}

Not applicable.

\section{Competing interests}

The authors declare that they have no competing interests.

\section{References}

1. Markman M: Current standards of care for chemotherapy of optimally cytoreduced advanced epithelial ovarian cancer. Gynecol Oncol 131: 241-245, 2013.

2. Pliarchopoulou K and Pectasides D: Epithelial ovarian cancer: Focus on targeted therapy. Crit Rev Oncol Hematol 79: 17-23, 2011.

3. He S, Niu G, Shang J, Deng Y, Wan Z, Zhang C, You Z and Shen H: The oncogenic Golgi phosphoprotein 3 like overexpression is associated with cisplatin resistance in ovarian carcinoma and activating the $\mathrm{NF}-\kappa \mathrm{B}$ signaling pathway. J Exp Clin Cancer Res 36: 137, 2017.

4. Roberts CM, Tran MA, Pitruzzello MC, Wen W, Loeza J, Dellinger TH, Mor G and Glackin CA: TWIST1 drives cisplatin resistance and cell survival in an ovarian cancer model, via upregulation of GAS6, L1CAM, and Akt signalling. Sci Rep 6: 37652, 2016. 
5. Roseman S: Reflections on glycobiology. J Biol Chem 276 41527-41542, 2001.

6. Iwamori M, Tanaka K, Kubushiro K, Lin B, Kiguchi K, Ishiwata I, Tsukazaki K and Nozawa S: Alterations in the glycolipid composition and cellular properties of ovarian carcinoma-derived RMG-1 cells on transfection of the $\alpha 1,2$-fucosyltransferase gene. Cancer Sci 96: 26-30, 2005.

7. Zhao Y, Lin B, Hao YY, Yan LM, Liu JJ, Zhu LC and Zhang SL: The effects of Lewis(y) antigen content on drug resistance to carboplatin in ovarian cancer line RMG-I. Prog Biochem Biophys 35: 1175-1182, 2008.

8. Zhang F, Liu J, Lin B, Liu Q, Zhao Y, Zhu L, Hao Y, Zhang S and Iwamori M: Increase in docetaxel-resistance of ovarian carcinoma-derived RMG-1 cells with enhanced expression of Lewis Y antigen. Int J Mol Sci 12: 7323-7334, 2011.

9. Liu J, Lin B, Hao Y, Qi Y, Zhu L, Li F, Liu D, Cong J, Zhang S and Iwamori M: Lewis y antigen promotes the proliferation of ovarian carcinoma-derived RMG-I cells through the PI3K/Akt signaling pathway. J Exp Clin Cancer Res 28: 154, 2009.

10. Hu Z, Gao J, Zhang D, Liu Q, Yan L, Gao L, Liu J, Liu D, Zhang S and Lin B: High expression of Lewis y antigen and CD44 is correlated with resistance to chemotherapy in epithelial ovarian cancers. PLoS One 8: e57250, 2013.

11. Gao J, Hu Z, Liu J, Liu D, Wang Y, Cai M, Zhang D, Tan M and Lin B: Expression of CD147 and Lewis y antigen in ovarian cancer and their relationship to drug resistance. Med Oncol 31: 920, 2014.

12. Zhu LC, Gao J, Hu ZH, Schwab CL, Zhuang HY, Tan MZ, Yan LM, Liu JJ, Zhang DY and Lin B: Membranous expressions of Lewis y and CAM-DR-related markers are independent factors of chemotherapy resistance and poor prognosis in epithelial ovarian cancer. Am J Cancer Res 5: 830-843, 2015.

13. Hu Z, Gao S, Gao J, Hou R, Liu C, Liu J, Li B, Liu D, Zhang S and Lin B: Elevated levels of Lewis y and integrin $\alpha 5 \beta 1$ correlate with chemotherapeutic drug resistance in epithelial ovarian carcinoma. Int J Mol Sci 13: 15588-15600, 2012.

14. Livak KJ and Schmittgen TD: Analysis of relative gene expression data using real-time quantitative PCR and the 2(- $\Delta \Delta \mathrm{C}(\mathrm{T}))$ method. Methods 25: 402-408, 2001.

15. Zhu L, Hu Z, Liu J, Gao J and Lin B: Gene expression profile analysis identifies metastasis and chemoresistance-associated genes in epithelial ovarian carcinoma cells. Med Oncol 32: 426, 2015.

16. Iwamori M, Iwamori Y, Kubushiro K, Ishiwata I and Kiguchi K: Characteristic expression of Lewis-antigenic glycolipids in human ovarian carcinoma-derived cells with anticancer drug-resistance. J Biochem 141: 309-317, 2007.

17. Madjd Z, Parsons T, Watson NF, Spendlove I, Ellis I and Durrant LG: High expression of Lewis $\mathrm{y} / \mathrm{b}$ antigens is associated with decreased survival in lymph node negative breast carcinomas. Breast Cancer Res 7: R780-R787, 2005.

18. Gao L, Yan L, Lin B, Gao J, Liang X, Wang Y, Liu J, Zhang S and Iwamori M: Enhancive effects of Lewis y antigen on CD44-mediated adhesion and spreading of human ovarian cancer cell line RMG-I. J Exp Clin Cancer Res 30: 15, 2011.

19. Yan LM, Lin B, Zhu LC, Hao YY, Qi Y, Wang CZ, Gao S, Liu SC, Zhang SL and Iwamori M: Enhancement of the adhesive and spreading potentials of ovarian carcinoma RMG-1 cells due to increased expression of integrin alpha5beta1 with the Lewis Y-structure on transfection of the alpha1,2-fucosyltransferase gene. Biochimie 92: 852-857, 2010

20. Tan M, Zhu L, Zhuang H, Hao Y, Gao S, Liu S, Liu Q, Liu D, Liu J and Lin B: Lewis $Y$ antigen modified CD47 is an independent risk factor for poor prognosis and promotes early ovarian cancer metastasis. Am J Cancer Res 5: 2777-2787, 2015.
21. Gao S, Liu Q, Wang X, Lin B and Zhang S: Effects of Lewis Y antigen on the gene expression of multiple drug resistanceassociated proteins in human ovarian cancer RMG-I-H cells. Med Oncol 27: 960-967, 2010.

22. Wang C, Yan L, Wang Y, Lin B, Liu S, Li Q, Gao L, Zhang S and Iwamori M: Overexpression of Lewis(y) antigen protects ovarian cancer RMG-1 cells from carboplatin-induced apoptosis by the upregulation of Topo-I and Topo-II $\beta$. Anat Rec (Hoboken) 294: 961-969, 2011.

23. Wang XQ, Sun P, O'Gorman M, Tai T and Paller AS: Epidermal growth factor receptor glycosylation is required for ganglioside GM3 binding and GM3-mediated suppression [correction of suppresion] of activation. Glycobiology 11: 515-522, 2001.

24. Liu JJ, Lin B, Hao YY, Li FF, Liu DW, Qi Y, Zhu LC, Zhang SL and Iwamori M: Lewis(y) antigen stimulates the growth of ovarian cancer cells via regulation of the epidermal growth factor receptor pathway. Oncol Rep 23: 833-841, 2010.

25. Li FF, Liu JJ, Liu DW, Lin B and Hao YY: Cong JP, Zhu LC, Gao S, Zhang SL and Iwamori M: Lewis Y regulates signaling molecules of the transforming growth factor $\beta$ path way in ovarian carcinoma derived RMG-I cells. Int J Oncol 40: 1196-1202, 2012.

26. Zhuang H, Gao J, Hu Z, Liu J, Liu D and Lin B: Co-expression of Lewis y antigen with human epididymis protein 4 in ovarian epithelial carcinoma. PLoS One 8: e68994, 2013.

27. Lee T, Guo K, Li S, Qin S and Liu S: Effects of ANXA4 on cell adhesive ability and expression of adhesion-related genes in hepatocellular carcinoma MHCC97H cell line. J Clin Trials 31: 588-591, 2013.

28. Matsuzaki S, Serada S, Morimoto A, Ueda Y, Yoshino K, Kimura T and Naka T: Annexin A4 is a promising therapeutic target for the treatment of platinum-resistant cancers. Expert Opin Ther Targets 18: 403-414, 2014.

29. Choi CH, Sung CO, Kim HJ, Lee YY, Song SY, Song T, Kim J, Kim TJ, Lee JW, Bae DS, et al: Overexpression of Annexin A4 is associated with chemoresistance in papillary serous adenocarcinoma of the ovary. Hum Pathol 44: 1017-1023, 2013.

30. Lin LL, Huang HC and Juan HF: Revealing the molecular mechanism of gastric cancer marker Annexin A4 in cancer cell proliferation using exon arrays. PLoS One 7: e44615, 2012.

31. Kelly MP, Lee FT, Tahtis K, Smyth FE, Brechbiel MW and Scott AM: Radioimmunotherapy with alpha-particle emitting 213Bi-C-functionalized trans-cyclohexyl-diethylenetriaminepentaacetic acid-humanized 3S193 is enhanced by combination with paclitaxel chemotherapy. Clin Cancer Res 13: 5604s-5612s, 2007.

32. Baldus SE, Mönig SP, Zirbes TK, Thakran J, Köthe D, Köppel M, Hanisch FG, Thiele J, Schneider PM, Hölscher AH, et al: Lewis(y) antigen (CD174) and apoptosis in gastric and colorectal carcinomas: Correlations with clinical and prognostic parameters. Histol Histopathol 21: 503-510, 2006.

33. Azuma Y, Ito M, Taniguchi A and Matsumoto K: Expression of cell surface Lewis X and Y antigens and FUT4 mRNA is increased in Jurkat cells undergoing apoptosis. Biochim Biophys Acta 1672: 157-163, 2004.

34. Azuma Y, Kurusu Y, Sato H, Higai K and Matsumoto K: Increased expression of Lewis $\mathrm{X}$ and $\mathrm{Y}$ antigens on the cell surface and FUT 4 mRNA during granzyme B-induced Jurkat cell apoptosis. Biol Pharm Bull 30: 655-660, 2007.

35. Gao N, Liu J, Liu D, Hao Y, Yan L, Ma Y, Zhuang H, Hu Z, Gao J, Yang Z, et al: c-Jun transcriptionally regulates alpha 1, 2-fucosyltransferase 1 (FUT1) in ovarian cancer. Biochimie 107: 286-292, 2014. 